\title{
Voltage-Controlled ON-OFF Ferromagnetism at Room Temperature in a Single Metal Oxide Film
}

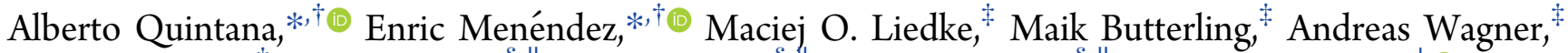 \\ Veronica Sireus, ${ }^{\dagger}$ Pau Torruella, ${ }^{\S, \|}$ Sònia Estradé, ${ }^{\S, \|}$ Francesca Peiró, ${ }^{\S, \|}$ Jolien Dendooven, ${ }^{\perp}(\mathbb{0}$

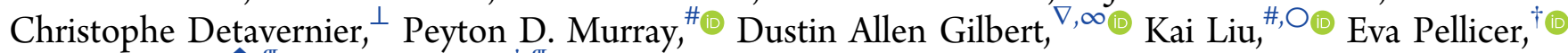 \\ Josep Nogues, $\rightarrow \mathbb{I}$ and Jordi Sort ${ }^{\dagger}, \mathbb{I}_{0}$ \\ ${ }^{\dagger}$ Departament de Física, Universitat Autònoma de Barcelona, E-08193 Cerdanyola del Vallès, Spain \\ ${ }^{\ddagger}$ Institute of Radiation Physics, Helmholtz-Center Dresden-Rossendorf, Dresden 01328, Germany \\ ${ }^{\S}$ LENS-MIND, Departament d'Enginyeria Electrònica i Biomèdica, Universitat de Barcelona, 08028 Barcelona, Spain \\ "Institute of Nanoscience and Nanotechnology (IN2UB), Universitat de Barcelona, 08028 Barcelona, Spain \\ ${ }^{\perp}$ Department of Solid State Sciences, CoCooN, Ghent University, Krijgslaan 281/S1, 9000 Ghent, Belgium \\ \#Physics Department, University of California, Davis, California 95616, United States \\ ${ }^{\nabla}$ NIST Center for Neutron Research, National Institute of Standards and Technology, Gaithersburg, Maryland 20899, United States \\ ODepartment of Physics, Georgetown University, Washington, D.C. 20057, United States \\ Catalan Institute of Nanoscience and Nanotechnology (ICN2), CSIC and BIST, Campus UAB, Bellaterra, E-08193 Barcelona, \\ Spain \\ II Institució Catalana de Recerca i Estudis Avançats (ICREA), Pg. Lluís Companys 23, E-08010 Barcelona, Spain \\ ${ }^{\infty}$ Materials Science and Engineering, University of Tennessee, Knoxville, Tennessee 37996, United States \\ Supporting Information
}

ABSTRACT: Electric-field-controlled magnetism can boost energy efficiency in widespread applications. However, technologically, this effect is facing important challenges: mechanical failure in strain-mediated piezoelectric/magnetostrictive devices, dearth of room-temperature multiferroics, or stringent thickness limitations in electrically charged metallic films. Voltage-driven ionic motion (magneto-ionics) circumvents most of these drawbacks while exhibiting interesting magnetoelectric phenomena. Nevertheless, magneto-ionics typically requires heat treatments and multicomponent heterostructures. Here we report on the electrolytegated and defect-mediated $\mathrm{O}$ and $\mathrm{Co}$ transport in $\mathrm{a} \mathrm{Co}_{3} \mathrm{O}_{4}$ single layer which allows for room-temperature voltage-controlled $\mathrm{ON}-\mathrm{OFF}$ ferromagnetism (magnetic switch) via internal reduction/oxidation processes. Negative voltages partially reduce $\mathrm{Co}_{3} \mathrm{O}_{4}$ to $\mathrm{Co}$ (ferromagnetism: $\mathrm{ON}$ ),

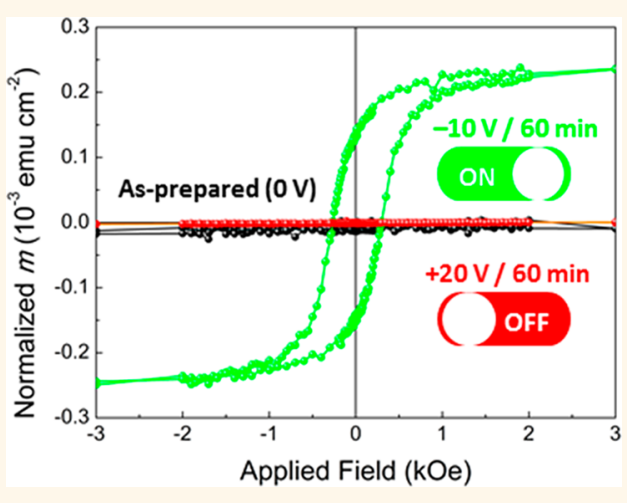
resulting in graded films including $\mathrm{Co}$ - and $\mathrm{O}$-rich areas. Positive bias oxidizes Co back to $\mathrm{Co}_{3} \mathrm{O}_{4}$ (paramagnetism: OFF). This electric-field-induced atomic-scale reconfiguration process is compositionally, structurally, and magnetically reversible and self-sustained, since no oxygen source other than the $\mathrm{Co}_{3} \mathrm{O}_{4}$ itself is required. This process could lead to electric-field-controlled device concepts for spintronics.

KEYWORDS: voltage control of magnetism, electrolyte, ion migration, magneto-ionics, on-off ferromagnetism

1 he search for energy-efficient materials and processes has become central to increasing industrial competitiveness, as well as to fulfilling environmental, health, and other societal expectations. Stable, strong, tunable, and nonvolatile voltage control of magnetism might allow for ultralow-power magnetic storage and spintronic devices. ${ }^{1-4}$ So far, magnetoelectric devices are controlled by electric currents, inherently involving a significant energy loss by Joule heating

Received: July 17, 2018

Accepted: September 26, 2018

Published: September 26, 2018 
a

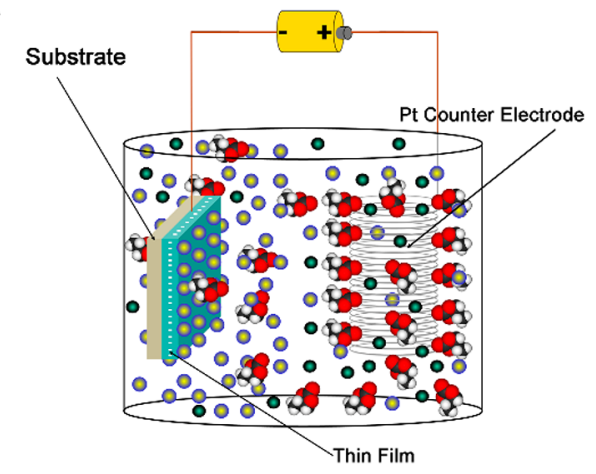

Nonoxidative Electrolyte

- $\mathrm{Na}^{+}$Ion $\quad \mathrm{OH}$ lon

8. Propylene Carbonate

C

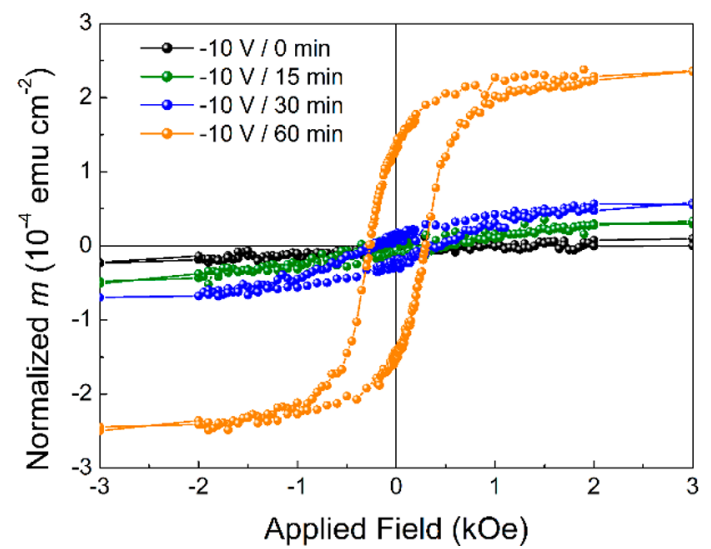

b

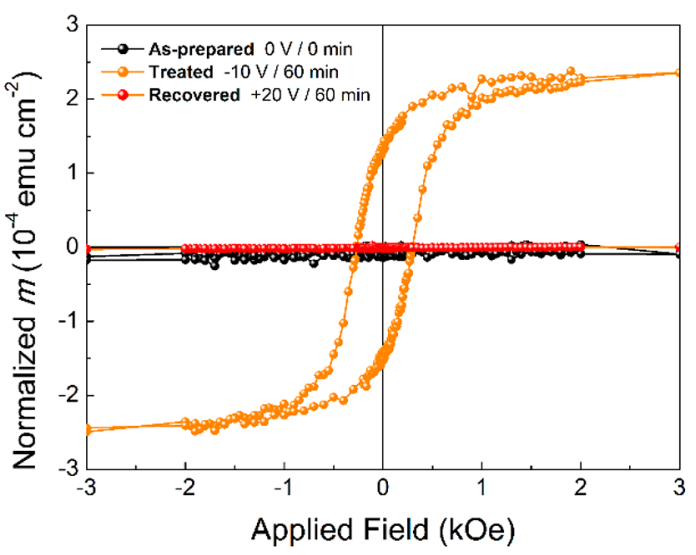

d

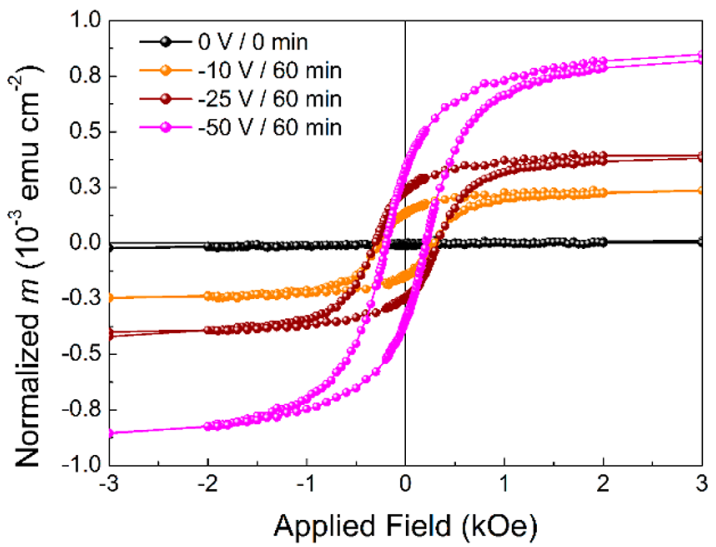

Figure 1. Voltage actuation and magnetic characterization by VSM. (a) Custom-made electrolytic cell used for the in situ voltage-applied VSM measurements. (b) Normalized magnetic moment (m) versus applied field VSM measurements of a pristine sample, after being treated at $-10 \mathrm{~V}$ for $60 \mathrm{~min}$ and after recovery having applied $+20 \mathrm{~V}$ for $60 \mathrm{~min}$. (c) VSM measurements corresponding to a sample subjected to $-10 \mathrm{~V}$ for $0,15,30$, and $60 \mathrm{~min}$. (d) VSM measurements corresponding to a sample subjected to decreasing negative voltages of 0 , -10 , -25 , and $-50 \mathrm{~V}$, applied during $60 \mathrm{~min}$ each. The measurements shown in (d) were recorded on the same sample. That is, upon negative biasing, the sample was brought to its pristine state with a recovery process with positive voltages. Specifically, the sample was recovered for $120 \mathrm{~min}$ at $+20,+50$, and $+100 \mathrm{~V}$ upon being treated for $60 \mathrm{~min}$ at $-10,-25$, and $-50 \mathrm{~V}$, respectively.

effect. The partial substitution of electric currents by electric fields to manipulate such devices (i.e., voltage-modulation of magnetism) would result in significant energy savings with an important cost reduction. ${ }^{3}$ Over the years, voltage control of magnetism has relied on several strategies, such as (i) development of single-phase multiferroic materials with intrinsic magnetoelectric coupling, ${ }^{5,6}$ (ii) inverse magnetostriction effects in epitaxial piezoelectric/ferromagnetic heterostructures, $^{6-9}$ or (iii) electric charge accumulation in dielectric/ferromagnetic condenser-like structures. ${ }^{10-14}$ Unfortunately, implementation of these materials in real devices is hampered by several factors. Single-phase multiferroics are scarce, are usually operative only at low temperatures, and still exhibit rather weak magnetoelectric coupling; artificial strainmediated piezoelectric/magnetostrictive heterostructures are prone to mechanical fatigue and thus have a limited endurance; finally, due to the subnanometric electric-field screening length in metallic ferromagnetic materials, electric charge accumulation effects are typically observed only in ultrathin films $(<2$ $\mathrm{nm}$ ) which are very sensitive to oxidation. Moreover, most of these approaches typically render volatile magnetic effects which disappear upon voltage removal.

Recently, voltage-induced ionic motion in magnetic materials (magneto-ionics) ${ }^{15-18}$ has attracted huge interest, since this phenomenon may allow for an electrical modulation of magnetism to an extent never attained by any other magnetoelectric means. Magneto-ionics results in a paradigm since it is not limited to the electric-field screening length and it can therefore be relevant in relatively thick films. However, in most previous magneto-ionics studies, voltage needs to be applied at high temperature since ion migration is a thermally activated process. ${ }^{15,16,18}$ Furthermore, the material of interest typically needs to be in contact with $\mathrm{Gd}_{2} \mathrm{O}_{3}$ or $\mathrm{HfO}_{2}$ layers, which act as ion reservoirs by accepting or donating oxygen ions depending on the voltage polarity. ${ }^{15,17,18}$ So far, the reported magneto-ionic effects are mostly limited to changes in coercivity, ${ }^{16-18}$ anisotropy easy axis, ${ }^{15,17}$ or exchange bias. ${ }^{19}$

Electrolyte gating (in particular, the use of electric double layers, EDL) allows for the generation of very large electric fields (due to the $<1 \mathrm{~nm}$ EDL thickness) capable of inducing $\mathrm{O}^{2-}$ migration in some oxides. This effect has been exploited to 

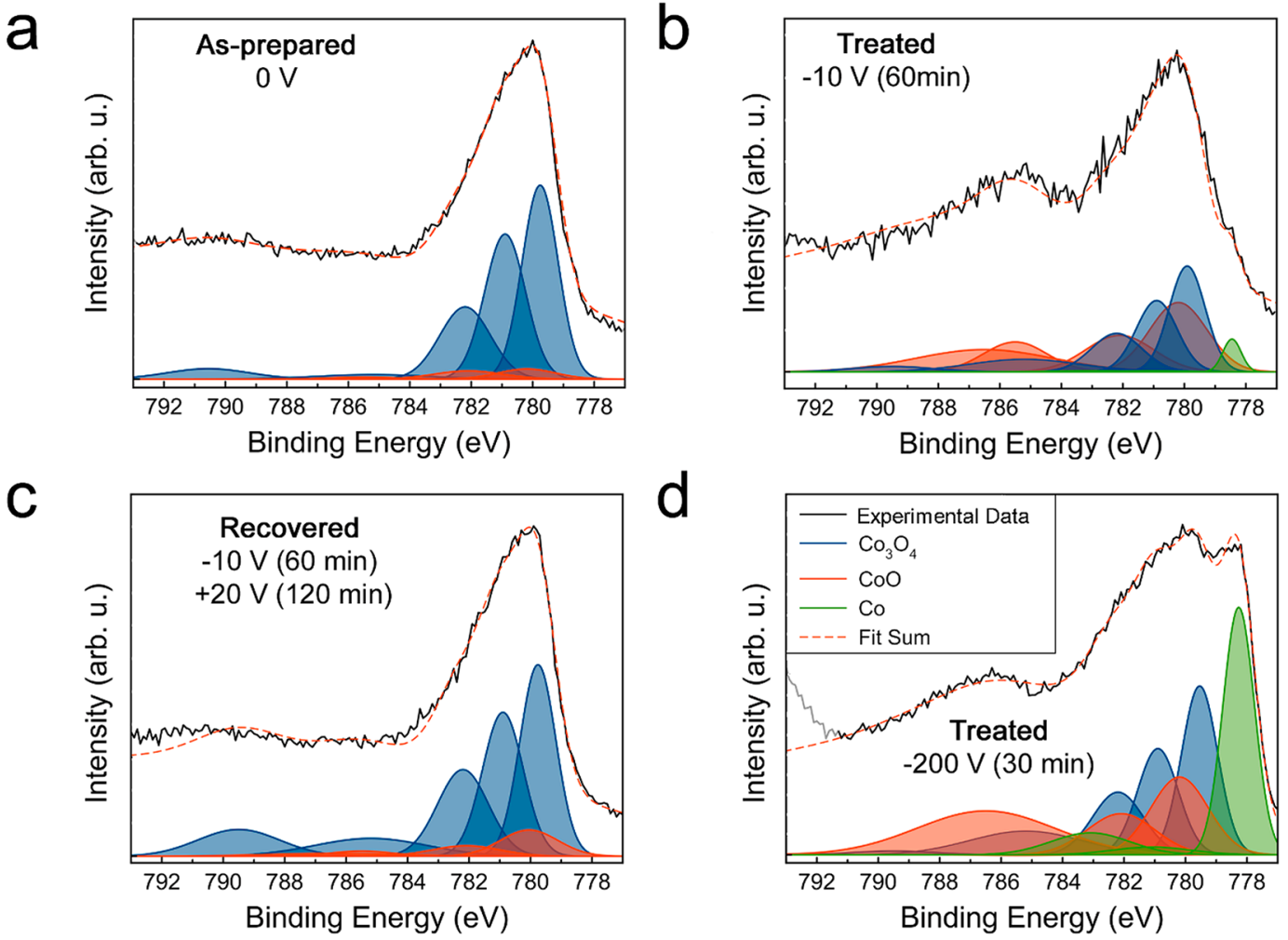

Figure 2. Compositional characterization by XPS. XPS core level Co $2 p$ spectrum of (a) the as-prepared sample, (b) the same sample upon $-10 \mathrm{~V}$ for $60 \mathrm{~min}$, and (c) the sample in (b) after applying $+20 \mathrm{~V}$ for $120 \mathrm{~min}$ and (d) another sample after being treated at $-200 \mathrm{~V}$ for 30 min. Spectra are deconvoluted taking into account $\mathrm{Co}_{3} \mathrm{O}_{4}, \mathrm{CoO}$, and Co contributions.

sensitively tune the electrical properties of a variety of materials, eventually suppressing/inducing insulator-to-conductive phase transitions. ${ }^{20-25}$ However, to date, magnetoionic control of ON/OFF ferromagnetism at room temperature has remained elusive.

A drastic voltage-induced $\mathrm{ON}-\mathrm{OFF}$ ferromagnetism at room temperature has only been achieved by tuning the antiferromagnetic-to-ferromagnetic metamagnetic transition of $\mathrm{FeRh}^{12}$ or the superparamagnetic blocking temperature of $\mathrm{Ni}$ nanoparticles, ${ }^{26}$ in both cases mediated by the coupling to underlying ferroelectric substrates. High-temperature voltageassisted oxidation of ultrathin $(0.7 \mathrm{~nm})$ Co layers in contact with $\mathrm{GdO}_{x}$ underlayers has also been used to suppress magnetization. ${ }^{15}$ In turn, ON-OFF ferromagnetism at low temperatures was achieved in $\mathrm{La}_{0.74} \mathrm{Sr}_{0.26} \mathrm{MnO}_{3}$ films by electrochemical (pseudocapacitance) doping. ${ }^{27} \mathrm{~A}$ maximum of up to $70 \%$ change of the magnetization has been observed in $\mathrm{ZnFe}_{2} \mathrm{O}_{4}$ films at room temperature by electrochemistry-driven Li-ion exchange. ${ }^{28}$

Herein, we report on the room-temperature voltage-induced ionic motion of oxygen and cobalt in a single $100 \mathrm{~nm}$ thick polycrystalline paramagnetic $\mathrm{Co}_{3} \mathrm{O}_{4}$ film. Negative biasing tends to reduce $\mathrm{Co}_{3} \mathrm{O}_{4}$ to $\mathrm{Co}$, resulting in ferromagnetism, whereas, upon positive voltages, the process can be completely reversed, oxidizing Co back to $\mathrm{Co}_{3} \mathrm{O}_{4}$. The amount of $\mathrm{Co}$, and thus the induced ferromagnetism, scales with time and the applied bias. Part of the induced ferromagnetism is permanent, but can be fully erased by applying positive voltages. Nonstoichiometry, primarily determined by defects such as oxygen and cobalt vacancies and grain boundaries, circumvents the lack of heat assistance and allows for a significant voltagedriven ionic motion even at room temperature. ${ }^{29}$ This approach leads to the possibility to take advantage of internal redox processes in single-phase thick layers, without the need of secondary oxides acting as donor/acceptor of oxygen.

\section{RESULTS AND DISCUSSION}

To investigate the influence of electric fields on the magnetic properties, the $\mathrm{Co}_{3} \mathrm{O}_{4}$ films, prepared by plasma-enhanced atomic layer deposition ${ }^{30,31}$ (see Methods), were subjected to voltage for a given period of time using a homemade electrolytic cell, as sketched in Figure 1a (i.e., electrolytegating). ${ }^{13,32}$ The voltage was then switched off and, immediately after, in-plane vibrating sample magnetometer (VSM) measurements were recorded in situ inside the electrolyte. An anhydrous liquid media (propylene carbonate with $\mathrm{Na}^{+}$solvated species) was used as electrolyte for two purposes: (i) to control the surroundings of the $\mathrm{Co}_{3} \mathrm{O}_{4}$ with a nonoxidizing medium, and (ii) to be able to maximize electric fields while applying low and moderate voltages. In this configuration, the $\mathrm{Co}_{3} \mathrm{O}_{4}$ film is used as a working electrode. Voltage is applied between the film electrode and a Pt counterelectrode (see Figure S1).

As voltage is increased, ions in the electrolyte solution diffuse toward the surface of the electrode forming the EDL which leads to strong electric fields on the order of hundreds of $\mathrm{MV} / \mathrm{cm} .{ }^{13}$ As can be seen in Figure $1 \mathrm{~b}$, the pristine $\mathrm{Co}_{3} \mathrm{O}_{4}$ sample shows no ferromagnetic behavior in agreement with its paramagnetic nature at room temperature. Conversely, after subjecting the sample to $-10 \mathrm{~V}$ for $60 \mathrm{~min}$, the measurements show a clear hysteresis loop, evidencing ferromagnetism. This generated ferromagnetism can be fully suppressed (and, thus, the process reversed) by applying a positive voltage of $20 \mathrm{~V}$ for $60 \mathrm{~min}$. It might be anticipated that this reversible 
phenomenon could be explained in a magneto-ionic framework through voltage-driven $\mathrm{O}^{2-}$ transport, giving rise to reduction and oxidation (i.e., redox) processes which are polarity dependent. For a given voltage (i.e., $-10 \mathrm{~V}$ ), Figure 1c shows the VSM measurements as a function of time. Further, Figure 1d shows the voltage-dependence of the hysteresis loops for a fixed biasing time (i.e., $60 \mathrm{~min}$ ). The magnetic moment scales with time and, in a more pronounced manner, with voltage, indicating that the underlying atomic mechanisms probably rely on voltage-activated diffusion effects. The measured magnetic signal after applying $-50 \mathrm{~V}$ is equivalent to a metallic $6 \mathrm{~nm}$ thick Co film. A single sample was used to investigate the voltage-dependence of the magnetic properties when subjecting the system to negative biases (Figure 1d), aimed at shedding light on the endurance characteristics of the system. The sample was first subjected to $-10 \mathrm{~V}$ for $60 \mathrm{~min}$ and, then, the voltage was switched off and, immediately after, a VSM measurement was recorded in situ inside the electrolyte. To recover the pristine state, the sample was subjected to +20 $\mathrm{V}$ for $120 \mathrm{~min}$. When treating the sample for $60 \mathrm{~min}$ at -25 and $-50 \mathrm{~V}$, the pristine state was recovered upon subjecting the sample for $120 \mathrm{~min}$ at +50 and $+100 \mathrm{~V}$, respectively. Treatments up to $-200 \mathrm{~V}$ were also carried out to maximize the effects and to subsequently perform a detailed structural characterization of the samples. As shown in Figure S2, upon removal of the $-200 \mathrm{~V}$ (applied for $30 \mathrm{~min}$ ), the magnetic moment relaxes following an exponential decay but a clear ferromagnetic signal remains (even for several months). Note that the hysteresis loop recorded immediately after such a high negative applied voltage is open (Figure S2a) since the magnetic moment progressively decreases during the time needed to acquire the loop. However, once the film is relaxed, the loops close, as expected. Note that this relaxation effect is considerably weaker for lower applied electric fields. For example, the loops obtained after applying lower voltages for 60 min close completely at high applied magnetic fields (Figure 1d).

To shed light on the atomic mechanisms which take place under voltage actuation, compositional characterization of the top sample surface through X-ray photoelectron spectroscopy (XPS) was carried out (Figure 2). As shown in Figure 2a, the XPS spectrum of the pristine sample is consistent with a rather pure $\mathrm{Co}_{3} \mathrm{O}_{4}$ phase with traces of $\mathrm{CoO}$, while, upon treatment at $-10 \mathrm{~V}$ for $60 \mathrm{~min}$, metallic Co or a Co-rich phase is present at least at the sample surface. Hence, negative biases promote reduction from $\mathrm{Co}_{3} \mathrm{O}_{4}$ to $\mathrm{CoO}$ and from $\mathrm{CoO}$ to ferromagnetic Co (Figure 2b), in agreement with the magnetometry results. It should be mentioned that $\mathrm{CoO}$ may partially come from natural oxidation of Co since the XPS is performed ex-situ and, thus, the sample is exposed to air. In contrast to negative biases, positive voltages allow for the recovery of the $\mathrm{Co}_{3} \mathrm{O}_{4}$ phase, evidencing that $\mathrm{Co}$ tends to reoxidize (Figure 2c). This demonstrates the high reversibility of the process, which not only takes place magnetically but also compositionally, and further indicates that voltage-driven redox processes could be a plausible scenario. Figure $2 \mathrm{~d}$ (corresponding to an applied voltage of $-200 \mathrm{~V}$ for $30 \mathrm{~min}$ ) confirms that the amount of generated Co scales with the applied negative voltage in concordance with the magnetic results.

From a compositional viewpoint, it is clear that this ONOFF ferromagnetism can be electrically modulated by voltagedriven ion migration. However, further information on morphological and structural aspects is essential to determine the mechanisms which govern this ionic transport. Actually, $\mathrm{Co}_{3} \mathrm{O}_{4}$ is prone to exhibit vacancies ${ }^{33}$ and, in the case of bulk $\mathrm{Co}_{3} \mathrm{O}_{4}$, Co migration is vacancy-mediated (i.e., via $\mathrm{Co}^{3+}$ vacancies). ${ }^{34,35}$

For a more detailed structural characterization, three samples were investigated: an as-deposited film, a film treated at $-200 \mathrm{~V}$ for $30 \mathrm{~min}$ (where the effects of voltage are maximized), and a sample treated at $-50 \mathrm{~V}$ for $30 \mathrm{~min}$ and subsequently fully recovered (i.e., brought back to the paramagnetic state) by applying $+100 \mathrm{~V}$ for $60 \mathrm{~min}$. Full recovery from the sample treated at $-200 \mathrm{~V}$ would require positive voltages higher than the ones available in our setup, hence the recovery was investigated in a sample treated at -50 V. Scanning electron microscopy (Figure S3) and $\theta / 2 \theta$ X-ray diffraction (Figure $S 4$ and Table S1) indicate that the ion migration mechanism is, at least partially, grain-boundarymediated (see the Supporting Information).

To further investigate the structure of the samples, cross section lamellae of the pristine and treated samples were prepared (Figure S5). The cross sections reveal the fully dense appearance of the films and confirm their average thickness of around $100 \mathrm{~nm}$. High resolution transmission electron microscopy (HRTEM) was carried out in a region close to the as-deposited sample surface, as indicated by the green square in Figure S5a (see Figure 3a). The corresponding fast Fourier transform (FFT) (Figure $3 \mathrm{~b}$ ) shows diffraction signal arising mainly from $\mathrm{Co}_{3} \mathrm{O}_{4}$ and a small contribution from $\mathrm{CoO}$ (Fm3m) (Table S2). Conversely, the sample treated at $-200 \mathrm{~V}$
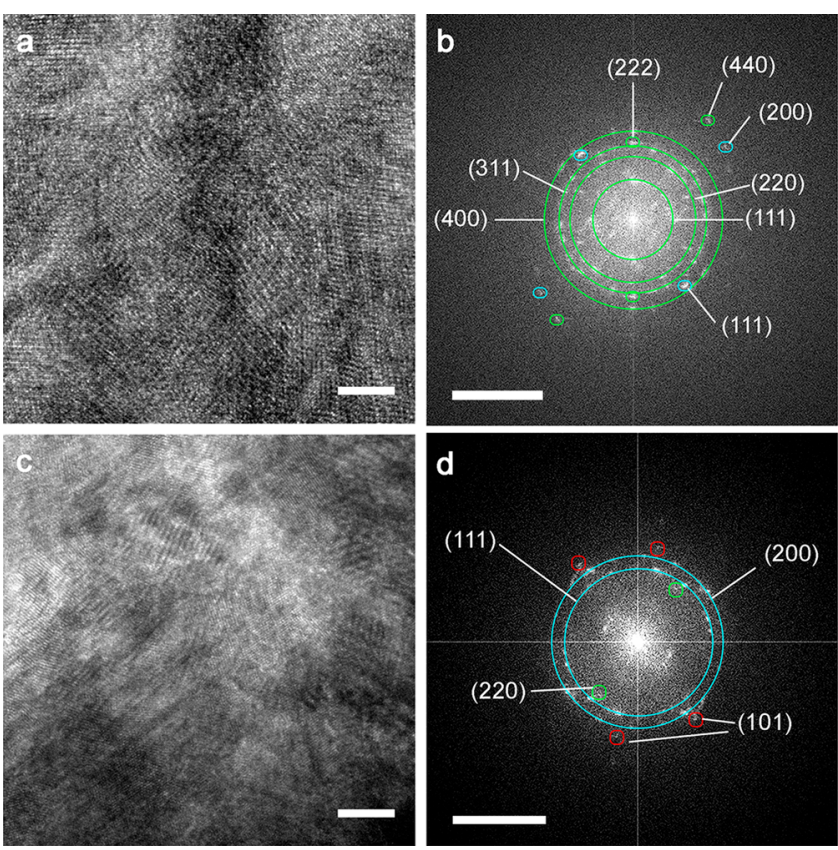

Figure 3. Structural characterization by HRTEM of the top regions of the films. ( $a, b)$ HRTEM image and corresponding fast Fourier transform of an untreated sample, respectively. (c, d) HRTEM image and corresponding fast Fourier transform of a sample treated at $-200 \mathrm{~V}$ for $30 \mathrm{~min}$, respectively. The scale bars in (a) and (c) correspond to $5 \mathrm{~nm}$, while those in (b) and (d) correspond to $5 \mathrm{~nm}^{-1}$. The main diffraction spots have been labeled and indexed (see Tables S2 and S3). In green, blue, and red, reflections corresponding to $\mathrm{Co}_{3} \mathrm{O}_{4}$ (JCPDF 42-1467), $\mathrm{CoO}$ (JCPDF 431004), and HCP-Co (JCPDF 05-0727) are indicated. 
for 30 min shows, besides $\mathrm{Co}_{3} \mathrm{O}_{4}$ and $\mathrm{CoO}$, clear spots from hexagonal close-packed ( $\mathrm{HCP}$ ) $\mathrm{Co}$, in agreement with the XPS characterization (green square in Figure S5b, Figure 3c,d, and Table S3). This confirms that, for negative applied voltages, $\mathrm{O}$ migrates from $\mathrm{Co}_{3} \mathrm{O}_{4}$ and leaves behind metallic $\mathrm{Co}$ areas. Similar results were also obtained from regions closer to the $\mathrm{SiO}_{2}$ interface (see Figure S6, which correspond to the red squares in Figure S5), evidencing that, at such voltages, the process not only reduces the surface but also affects the whole $\mathrm{Co}_{3} \mathrm{O}_{4}$ film (Tables S4-S7). In Figure S7, a HCP-Co nanocrystallite is identified and highlighted in red. The small volume fraction of the generated HCP-Co metallic clusters (only about 6\%) and their nanocrystalline character (with sizes of only a few $\mathrm{nm}$ ), makes it virtually impossible to detect them by using laboratory X-rays (red curve in Figure S4). Finally, HRTEM further confirms that, upon positive biasing, the initial state is recovered (Figure S8).

To investigate the $\mathrm{Co}-\mathrm{O}$ distribution, energy-filtered transmission electron microscopy (EFTEM) imaging of the as-deposited, treated and recovered samples was performed. In the as-deposited sample (Figure $4 \mathrm{a}$ ), a rather homogeneous
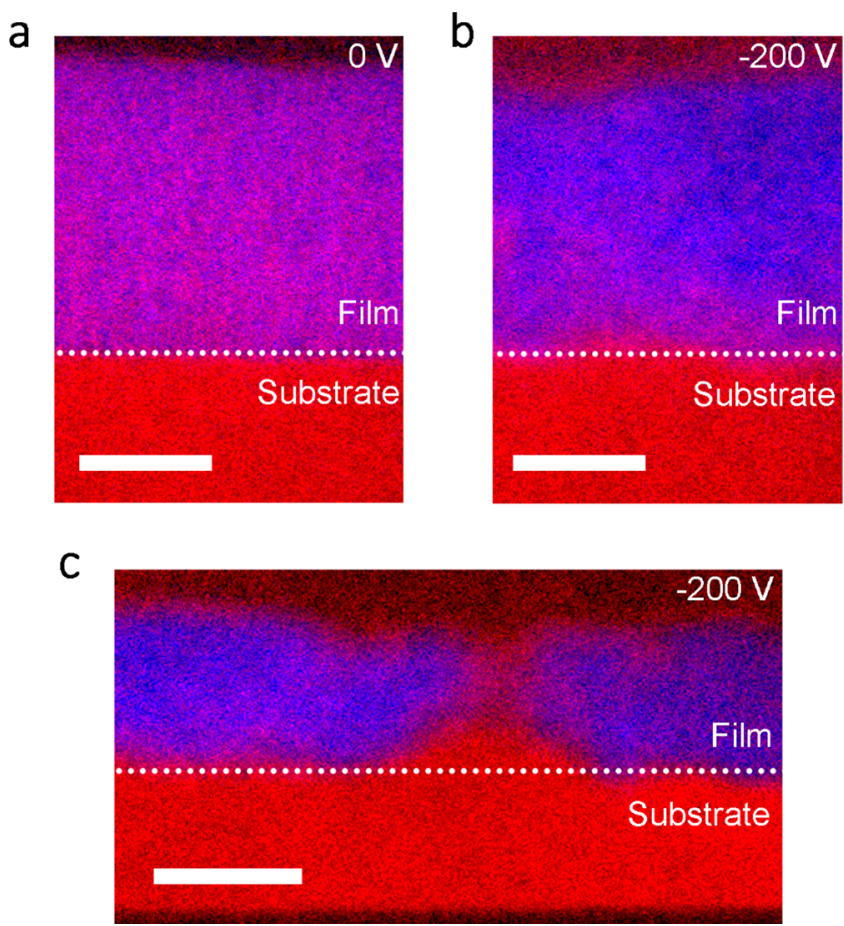

Figure 4. Depth-resolved structural and compositional characterization by EFTEM. EFTEM images corresponding to (a) the asgrown sample and $(b, c)$ two different regions of a negatively biased sample at $\mathbf{- 2 0 0} \mathrm{V}$ for $\mathbf{3 0} \mathrm{min}$. Scale bars correspond to $\mathbf{5 0}$ $\mathrm{nm}$ in (a) and (b), and to $100 \mathrm{~nm}$ in (c). Blue spots correspond to Co and red spots to $O$.

distribution of $\mathrm{Co}$ and $\mathrm{O}$ can be observed. In contrast, the presence of Co-rich and O-rich areas is clearly detected after negative biasing using $-200 \mathrm{~V}$ (Figure $4 \mathrm{~b}, \mathrm{c}$ ). Figure $4 \mathrm{~b}$ also reveals that $\mathrm{O}$ ions migrate toward the $\mathrm{SiO}_{2}$ interface following the electric field which is applied perpendicularly to the sample surface (see the way voltage is applied in Figure S1), while Co cations diffuse in opposite sense, resulting in a Co-rich top part and an O-rich bottom layer. Interestingly, O-rich channels are also observed (Figure 4c). These channels could act as diffusion paths and allow for a large incorporation of $\mathrm{O}$, to compensate for the formation of metallic Co areas. Upon recovery, $\mathrm{Co}$ and $\mathrm{O}$ redistribute and the sample becomes compositionally homogeneous again (Figure S9).

To locally quantify the $\mathrm{Co}-\mathrm{O}$ distribution, $\mathrm{Co}$ and $\mathrm{O}$ electron energy loss spectroscopy (EELS) mappings were conducted. As shown in Figure $5 \mathrm{a}-\mathrm{c}$, both $\mathrm{Co}$ and $\mathrm{O}$ are homogeneously distributed in the as-grown sample, with Co and $\mathrm{O}$ atomic percentages corresponding rather well to those of stoichiometric $\mathrm{Co}_{3} \mathrm{O}_{4}$ (i.e., 42.9 and 57.1 atom \% Co and $\mathrm{O}$, respectively). In contrast to the as-grown sample, the HAADFSTEM images of two different regions of the sample biased at $-200 \mathrm{~V}$ suggest strong composition inhomogeneities. The corresponding EELS mappings reveal the presence of Co-rich and $\mathrm{O}$-rich areas, with compositional variations ranging from almost pure $\mathrm{Co}$ to O-rich regions with $\mathrm{Co}_{20} \mathrm{O}_{80}$ (atom \%) (Figure 5e,f,h,i). These regions (i.e., Figure $5 \mathrm{~d}, \mathrm{~g}$ ) are representative of two main ion migration processes that occur in the system when a negative voltage is applied. The Co and $\mathrm{O}$ EELS mappings (Figure 5e,f) of the region corresponding to Figure $5 \mathrm{~d}$ evidence that, upon negative biasing, oxygen ion migration is promoted along channels which act as diffusion paths, leaving aside Co-rich areas. This is in agreement with the EFTEM characterization of Figure 4c. These channels are about $40 \mathrm{~nm}$ wide and appear sporadically (for instance, along an approximately $400 \mathrm{~nm}$ section of the sample, only one diffusion channel is observed in Figure 4c). As can be seen in Figure S10, the pristine sample tends to exhibit a columnar-shaped morphology along its width suggesting that grain boundaries, where diffusion is usually enhanced with respect to the inner parts of the grains, may sometimes contribute to the formation of these channels which act as diffusion paths for $\mathrm{O}$. As can be seen in Figure S11, which shows a high resolution TEM image of one of these Orich channels, these paths are of highly nanostructured nature or even partly amorphous-like, allowing for an enhanced $\mathrm{O}$ diffusion and a large incorporation of $\mathrm{O}$ as it happens in amorphous cobalt oxide phases which exhibit a high catalytic response. $^{36,37}$ This is reminiscent of the proposed mechanisms for resistive switching in some oxide materials. ${ }^{38}$ Additionally, Figure $5 \mathrm{~g}$ and the corresponding Co and O EELS mappings (Figure $5 \mathrm{~h}, \mathrm{i}$ ) represent a second migration process, which is characterized by a more uniform migration of ions along the depth of the film. Oxygen ions homogeneously diffuse toward the $\mathrm{SiO}_{2}$ interface (i.e., from the top to the bottom) following the electric field which is applied perpendicularly to the sample surface (see the way voltage is applied in Figure S1), while Co cations diffuse in the opposite sense, resulting in a Co-rich top part and an O-rich bottom layer. This is in agreement with the EFTEM characterization of Figure $4 \mathrm{~b}$. These results indicate that formation of HCP-Co is accompanied by an increase of the $\mathrm{O}$ content in $\mathrm{Co}_{3} \mathrm{O}_{4}$ (i.e., formation of cationic vacancies in the structure of $\mathrm{Co}_{3} \mathrm{O}_{4}$ ). Actually, it is known that $\mathrm{Co}_{3} \mathrm{O}_{4}$ can accommodate more oxygen than the stoichiometric composition in its structure, ${ }^{39}$ particularly under certain conditions of temperature and oxygen partial pressure. ${ }^{40}$ Here, such an effect is electric-field-induced. Similar results have been reported in electrolyte-gated $\mathrm{VO}_{2}$, where oxygen concentrates forming chains of edge-sharing $\mathrm{VO}_{6}$ octahedra. ${ }^{24}$ Analogous chains of dimeric-sharing $\mathrm{CoO}_{6}$ octahedra have also been reported in $\mathrm{CoO}_{x}$ nanoparticles. ${ }^{36} \mathrm{~A}$ reduction of the interplanar distances has been reported in O-rich $\mathrm{Co}_{3} \mathrm{O}_{4}$ fibers. ${ }^{38}$ The coexistence of stoichiometric with off-stoichiometric $\mathrm{Co}_{3-x} \mathrm{O}_{4+x}$ regions could be correlated with the asymmetry in the XRD peaks of the 

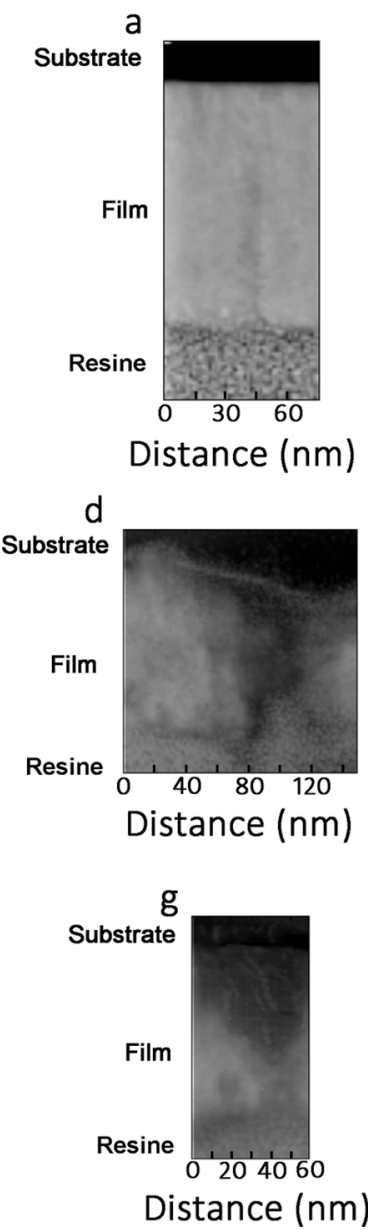

b

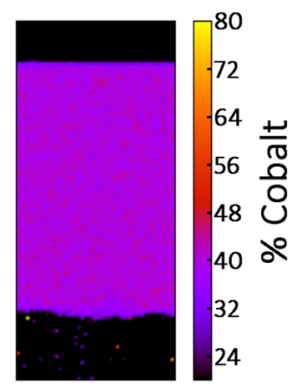

e

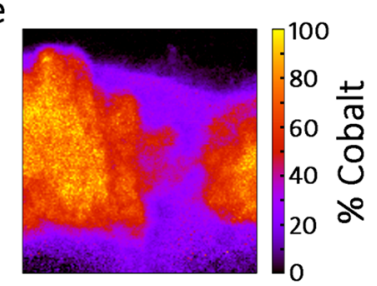

h

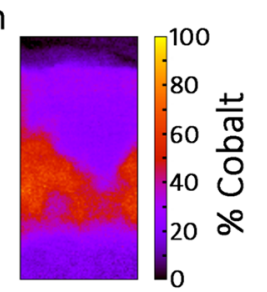

C

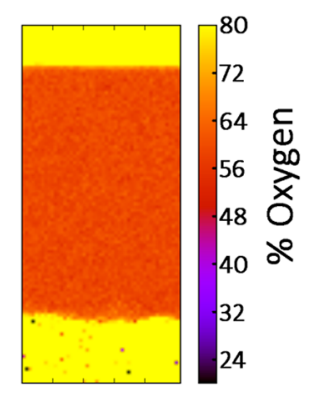

f

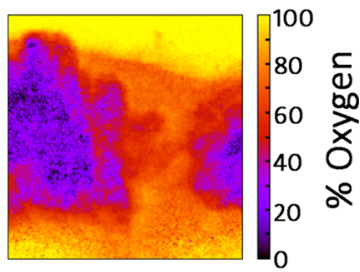

i

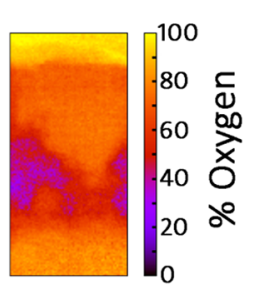

Figure 5. Structural and compositional characterization by high-angle annular dark-field scanning transmission electron microscopy HAADF-STEM and EELS. $(\mathrm{a}-\mathrm{c}),(\mathrm{d}-\mathrm{f})$, and $(\mathrm{g}-\mathrm{i})$ HAADF, Co, and $\mathrm{O}$ EELS mappings of the pristine sample, and two different regions of a sample negatively biased at $-200 \mathrm{~V}$ for $30 \mathrm{~min}$, respectively.

negatively biased sample (Figure S4). Anyhow, when negatively gating the system, no oxygen bubbling is observed, ruling out $\mathrm{O}_{2}$ formation. Assuming that no oxygen dissolves in the liquid electrolyte, $\mathrm{O}$ must redistribute along the sample while preserving charge neutrality. In contrast to crystalline materials, ${ }^{41}$ amorphous phases can promote $\mathrm{O}-\mathrm{O}$ bond coupling, $^{36}$ thus allowing for neutrally charged phases containing large amounts of oxygen as it happens in the observed O-rich diffusion channels which show compositions with $\mathrm{O}$ contents as large as 80 at. $\%$ (i.e., $\mathrm{Co}_{20} \mathrm{O}_{80}$ ).

To rule out spurious effects arising from $\mathrm{Na}^{+}$from the electrolyte or $\mathrm{Si}^{4+}$ from the substrate, energy-dispersive X-ray (EDX) spectroscopy and EELS spectra were acquired on the sample treated at $-200 \mathrm{~V}$ for $30 \mathrm{~min}$. Neither $\mathrm{Na}$ nor Si was detected (Figures S12-S14). The mechanism reported herein is thus different from previous works on lithiation of $\mathrm{ZnFe}_{2} \mathrm{O}_{4}$, $\mathrm{CuFe}_{2} \mathrm{O}_{4}$ and $\gamma-\mathrm{Fe}_{2} \mathrm{O}_{3}$ aiming at partially tuning their magnetization by electrochemical treatment in $1 \mathrm{M} \mathrm{LiPF}_{6}$ in ethylene carbonate and dimethyl carbonate solutions. ${ }^{28,42}$ Finally, Co and O EELS mappings were also performed for the fully recovered sample and, as expected, a homogeneous composition throughout the film close to stoichiometric $\mathrm{Co}_{3} \mathrm{O}_{4}$ was obtained (Figure $\mathrm{S} 15$ ).

Thus, the structural characterization clearly demonstrates that, in contrast to conventional magneto-ionic systems where there is a source/reservoir of oxygen to trigger the redox processes, ${ }^{15-18}$ in the here-prepared $\mathrm{Co}_{3} \mathrm{O}_{4}$ films, the magnetic switch is accomplished by a reversible atomic scale reconfiguration of the $\mathrm{O}$ and $\mathrm{Co}$ ions within the film itself.

Variable energy (VE) and coincidence Doppler broadening (cDB) positron annihilation spectroscopy (PAS) ${ }^{43,44}$ as well as positron annihilation lifetime spectroscopy (PALS) experiments were performed (see Methods and the Supporting Information) in an attempt to shed further light on the presence of negatively charged open volume defects (i.e., cationic Co vacancies, $\mathrm{Co}-\mathrm{O}$ vacancy complexes and grain boundaries) with depth-resolved resolution.

VE-PAS experiments (Figure S16) evidence a relative increase of the low electron momentum fraction, $S$ (directly proportional to the size and concentration of defects, ${ }^{45}$ see Supporting Information), after treatment using negative voltages, while $S$ concomitantly decreases again after positive biasing. This is in agreement with the increase of open volume defects for negatively biased samples, supporting the presence of channels for ionic O transport. Since VE-PAS cannot clearly distinguish between defects size and defect concentration, additional PALS and cDB-PAS investigations were performed.

The PALS spectra after background correction were deconvoluted using the nonlinearly least-squared-based package PALSfit fitting software ${ }^{46,47}$ into three discrete lifetime components, which directly evidence three different defect types (sizes) [see Figures $6 \mathrm{a}, \mathrm{b}$ and $\mathrm{S} 17 \mathrm{a}]$. The resulting 

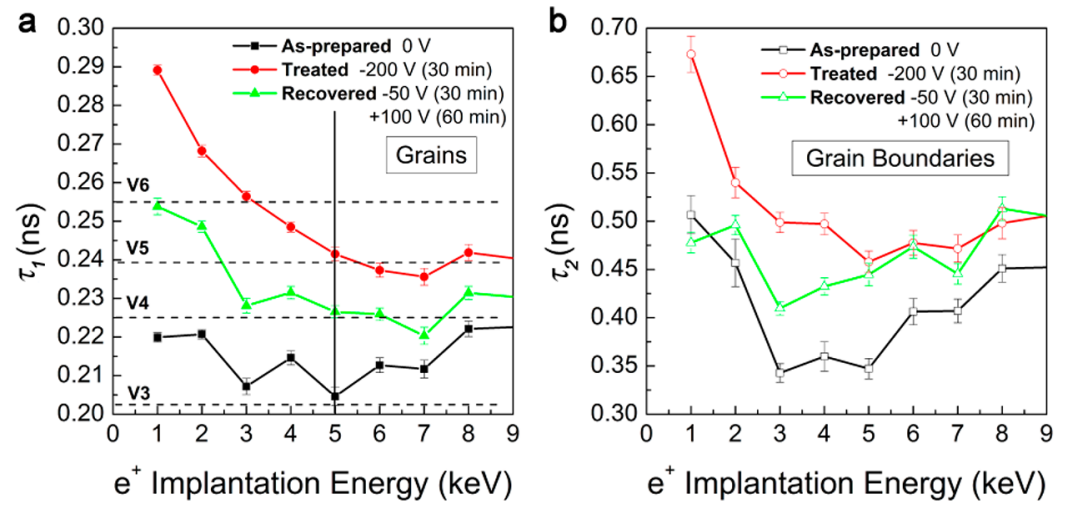
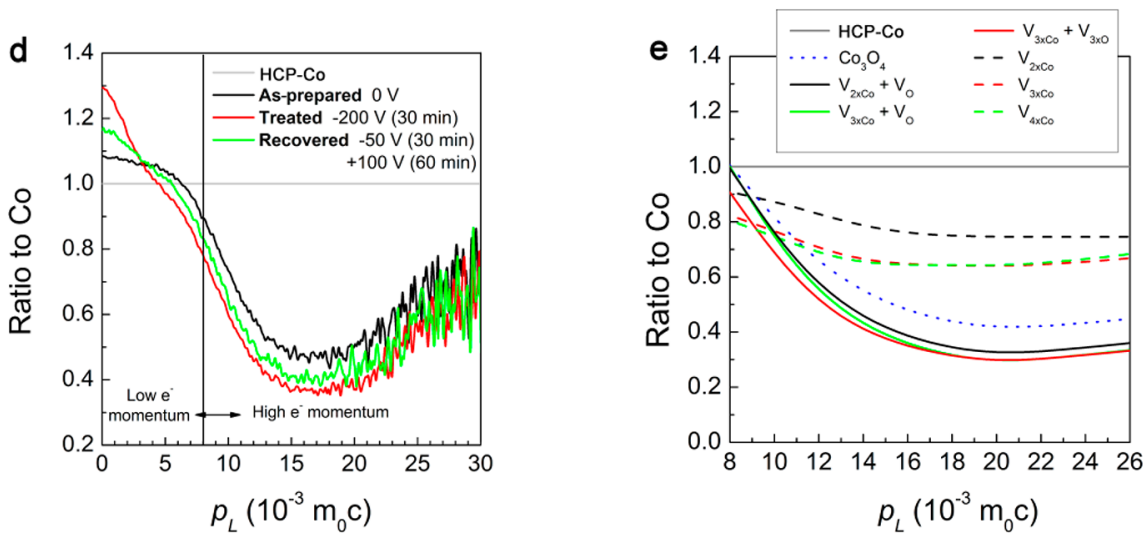

Figure 6. Positron annihilation spectroscopy characterization: $(a-c)$ Positron annihilation lifetime spectroscopy (PALS) analysis, where contributions of the annihilation signal from grains (a) and grain boundaries (b) are shown for as-grown (black), treated (red), and recovered (green) samples. The black vertical lines show an approximate position of the interface between the $\mathrm{Co}_{3} \mathrm{O}_{4} \mathrm{film}$ and the $\mathrm{SiO}_{2}$ buffer layer. The straight lines in a mark the lifetime of the vacancy complexes of different sizes: from trimers (V3) to clusters of six vacancies (V6). (c) Absolute intensity of the lifetime components. (d) Coincidence Doppler broadening ratio plots (normalization by a defect free Co reference) measured at $E_{\mathrm{p}}=3 \mathrm{keV}$, with a corresponding atomic superposition (ATSUP) simulation (e).

intensities reflect the concentration of each defect type (size) [Figures $6 \mathrm{c}$ and $\mathrm{S} 17 \mathrm{~b}$ ]. In general, positron lifetime scales with defects size (i.e., the larger the open volume, the longer it takes for positrons to be annihilated). ${ }^{44,47,48}$ The shortest lifetime component $\left(\tau_{1}<0.30 \mathrm{~ns}\right)$ represents vacancy clusters inside the grains, whereas the intermediate $\left(0.35<\tau_{2}<0.70 \mathrm{~ns}\right)$ and the longest $\left(1.5<\tau_{3}<4.0 \mathrm{~ns}\right)$ lifetime components indicate grain boundaries and larger voids (1-3 nm in diameter) contributions, respectively. The lifetime of small vacancy clusters $\left(\tau_{1}\right)$ is around $0.21 \mathrm{~ns}$ in the as-grown sample. This lifetime strongly increases (up to $\tau_{1} \approx 0.29 \mathrm{~ns}$ ) in the sample treated at $-200 \mathrm{~V}$ (indicating an increase of the average vacancy size), and then decreases down to about $0.23-0.25 \mathrm{~ns}$ in the recovered sample. The lifetime component $\tau_{2}$, representing grain boundary contributions, behaves similarly, which is in accordance with a $\mathrm{Co}$ and $\mathrm{O}$ ionic migration mechanism. Namely, during negative biasing, ionic motion is promoted at the grain boundaries and large open volume defects are left behind, so that disordered grain boundaries increase in size (in agreement with Figure S3c); positive biasing reverses the ionic migration and defects states are partially refilled. The contribution from the $\tau_{3}$ component within the films is below 1\% (see the Supporting Information); thus, it can be neglected.

In order to translate the obtained positron lifetime values to realistic defects sizes, additional cDB-PAS measurements were also conducted (Figure 6d) and compared with theoretical calculations by means of the so-called atomic superposition
(ATSUP) method ${ }^{49,50}$ (Figure 6e) [see Methods and the Supporting Information]. Electron momentum distributions, acquired at $E_{\mathrm{p}}=3 \mathrm{keV}$ and normalized to a Co defect-free reference spectrum, are presented in Figure $6 \mathrm{~d}$ for the asprepared, treated, and recovered samples, where annihilation events come from the film region only (see Figure S18). The low momentum part of the spectra, $p_{\mathrm{L}}<8 \times 10^{-3} m_{0} c$, is simply another representation of $S\left(E_{\mathrm{p}}=3 \mathrm{keV}\right)$ from Figure $S 16$. The high electron momentum part of the ratio plots, $p_{\mathrm{L}}>8 \times$ $10^{-3} m_{0} c$, shows a minimum at $p_{\mathrm{L}} \approx 16 \times 10^{-3} m_{0} c$ for all the samples, which is in qualitative agreement with the calculated curves from Figure 6e. The high momentum part is a fingerprint of the defect site atomic surrounding and, interestingly, it does not consist of pure HCP-Co phase but, instead, is in agreement with the calculations for mixed vacancies involving different amounts of $\mathrm{O}$ and $\mathrm{Co}$ atoms (Figure 6e). This does not exclude Co segregation, since positrons preferentially annihilate at the oxide phase (due to their higher affinity to oxides).

Table S8 shows the calculated positron lifetime values for different types of vacancy defects in HCP-Co and $\mathrm{Co}_{3} \mathrm{O}_{4}$. The results indicate that existence of small vacancy clusters, probably in the form of cobalt vacancy dimers (two vacancies), $\mathrm{V}_{2 x \mathrm{Co}}$, or trimers (three vacancies), $\mathrm{V}_{3 x \mathrm{Co}}$, coupled with an oxygen monovacancy (single vacancy), $\mathrm{V}_{\mathrm{O}}$ (where a lifetime higher than $0.2 \mathrm{~ns}$ is obtained). These configurations, compatible with our experiment, are indicated in bold in Table S8 (further details can be found in the Supporting 
Information). The experimental values of $\tau_{1}$ corresponding to the treated samples are still larger than the calculated value of $0.2526 \mathrm{~ns}$ which corresponds to six vacancies (see Figure 6a and Table S8) upon the assumption of a stoichiometric and crystalline $\mathrm{Co}_{3} \mathrm{O}_{4}$ phase in the simulations. This discrepancy is in concordance with the formation of highly nanostructured or even amorphous-like phases upon negative biasing.

\section{CONCLUSIONS}

In conclusion, voltage-driven $\mathrm{O}$ and $\mathrm{Co}$ redistribution has been demonstrated in $100 \mathrm{~nm}$ thick $\mathrm{Co}_{3} \mathrm{O}_{4}$ films through electrolyte-gating, allowing for the controlled generation and suppression of ferromagnetism. A negative voltage reduces $\mathrm{Co}_{3} \mathrm{O}_{4}$ to $\mathrm{Co}$ (ferromagnetism: $\mathrm{ON}$ ), whereas the process is reversed by applying a positive bias, aimed at oxidizing $\mathrm{Co}$ back to $\mathrm{Co}_{3} \mathrm{O}_{4}$ (paramagnetism: OFF). These gate-induced $\mathrm{O}$ and $\mathrm{Co}$ migrations are driven by mixed vacancy clusters as evidenced by positron annihilation spectroscopy. Ionic transport seems to be promoted at grain boundaries and is further assisted by the formation of diffusion channels that incorporate large amounts of $\mathrm{O}$. Part of the generated ferromagnetism is not transient but stable, although it can be easily erased by applying adequate positive voltage values. The process is selfsustained in the sense that no external source/sink of oxygen is required. Our approach also circumvents the need of thermally assisted ionic migration, i.e., the voltage-driven oxygen motion takes place at room temperature. Furthermore, our procedure could in principle be extended to room temperature oxidebased antiferromagnets (e.g., $\mathrm{NiO}$ ), allowing for an extra degree of freedom taking advantage of exchange bias effects.

\section{METHODS}

Sample Preparation. $\mathrm{Co}_{3} \mathrm{O}_{4}$ films were grown on thermally oxidized $\mathrm{Si}$ wafers (i.e., $\left.\mathrm{SiO}_{2}(100 \mathrm{~nm}) /\langle 100\rangle \mathrm{Si}(1 \mathrm{~mm})\right)$ by plasmaenhanced atomic layer deposition (PE-ALD) using bis(cyclopentadienyl)cobalt (CoCp2, STREM, min. 98\%) as Co precursor and $\mathrm{O}^{2}$ plasma as oxygen source. ${ }^{30}$ The depositions were performed in a home-built ALD reactor with a base pressure of $10^{-6}$ mbar. ${ }^{30,31}$ The container with the solid $\mathrm{CoCp}_{2}$ precursor and the tube to the reactor were heated to 80 and $100{ }^{\circ} \mathrm{C}$, respectively, to prevent deposition of the precursor in the tubes or valves. The $\mathrm{Co}_{3} \mathrm{O}_{4}$ layers were produced by alternate pulsing of $\mathrm{CoCp}_{2}$ and $\mathrm{O}_{2}$ plasma at a deposition temperature of $200{ }^{\circ} \mathrm{C}$. Argon was used as a carrier gas for the Co precursor. The ALD cycle consisted of $5 \mathrm{~s} \mathrm{CoCp_{2 }}$ exposure, 5 $\mathrm{s}$ of pumping, $5 \mathrm{~s}_{2}$ plasma exposure and $5 \mathrm{~s}$ of pumping. During the exposures, the pressure in the ALD chamber was in the $10^{-3} \mathrm{mbar}$ range. The growth rate was $0.5 \AA /$ cycle, and a total of 2000 ALD cycles were applied to obtain $100 \mathrm{~nm}$ thick $\mathrm{Co}_{3} \mathrm{O}_{4}$ films.

Structural and Compositional Measurements. Scanning electron microscopy (SEM) images were acquired using secondary electrons in a FEI Magellan 400L microscope operated at $20 \mathrm{kV}$. Transmission electron microscopy (TEM), scanning transmission electron microscopy/energy-dispersive X-ray spectroscopy (STEM/ EDX), and electron energy loss spectroscopy (EELS) were performed on a TECNAI F20 HRTEM/STEM microscope operated at $200 \mathrm{kV}$. Cross-sectional lamellae from the as-grown, treated $(-200 \mathrm{~V}$ for 30 $\mathrm{min})$, and recovered $(-50 \mathrm{~V}$ for $30 \mathrm{~min} /+100 \mathrm{~V}$ for $1 \mathrm{~h}$ ) samples were prepared by focused ion beam (FIB) and placed onto a Cu TEM grid.

$\theta / 2 \theta \mathrm{X}$-ray diffraction (XRD) patterns of the different samples were recorded on a Philips X'Pert Powder diffractometer with a Pixel1D detector in the $17^{\circ}-40^{\circ} 2 \theta$ range using $\mathrm{Cu} \mathrm{K} \alpha$ radiation with intensity $\left(\mathrm{Cu} \mathrm{K} \alpha_{2}\right) /$ intensity $\left(\mathrm{Cu} K \alpha_{1}\right)=0.5$. The structural parameters of $\mathrm{Co}_{3} \mathrm{O}_{4}$, such as crystallite size (i.e., average coherently diffracting domain) or lattice parameter were evaluated by fitting the
XRD patterns in the $18^{\circ}-20^{\circ} 2 \theta$ range using the MAUD Rietveld refinement program. ${ }^{51}$

Variable energy positron annihilation spectroscopy (VE-PAS) ${ }^{43,44}$ was used to investigate depth-resolved open volume defects at the Slow-Positron System of Rossendorf (SPONSOR) beamline, which provides monoenergetic but variable energy positron beam. Further details on this type of experiments, as well as on positron annihilation lifetime spectroscopy (PALS) and theoretical calculations using the atomic superposition (ATSUP) method are given in the Supporting Information.

Magnetic Characterization. Magnetic measurements were carried out at room temperature in a vibrating sample magnetometer from Micro Sense (LOT-Quantum Design), with a maximum applied magnetic field of $2 \mathrm{~T}$. Figure 1a illustrates the experimental setup used for the in situ magnetoelectric measurements. The sample was mounted in a homemade electrolytic cell filled with anhydrous propylene carbonate with $\mathrm{Na}^{+}$solvated species (i.e., nonoxidative media), and the magnetic properties were measured along the film plane after applying different voltages, using an external Agilent B2902A power supply, between the sample and the counter-electrode in a similar fashion of that presented in references. ${ }^{13,31}$ The $\mathrm{Na}^{+}$ solvated species in the electrolyte are aimed at reacting with any traces of water. The longitudinal magnetic signal was normalized to the area of the sample immersed in the electrolyte.

\section{ASSOCIATED CONTENT}

\section{S Supporting Information}

The Supporting Information is available free of charge on the ACS Publications website at DOI: 10.1021/acsnano.8b05407.

Experimental setup to carry out magnetoelectric measurements (i.e., VSM measurements under voltage application); further magnetoelectric measurements; further structural (SEM, TEM, HRTEM, XRD) and compositional (EFTEM, HAADF-STEM, and EELS) characterization; further analysis by PALS and VE-PAS techniques (PDF)

\section{AUTHOR INFORMATION}

\section{Corresponding Authors}

*E-mail: alberto.quintana@uab.cat.

*E-mail: enric.menendez@uab.cat.

ORCID ${ }^{\circ}$

Alberto Quintana: 0000-0002-9813-735X

Enric Menéndez: 0000-0003-3809-2863

Jolien Dendooven: 0000-0002-2385-3693

Peyton D. Murray: 0000-0003-0389-0611

Dustin Allen Gilbert: 0000-0003-3747-3883

Kai Liu: 0000-0001-9413-6782

Eva Pellicer: 0000-0002-8901-0998

Jordi Sort: 0000-0003-1213-3639

\section{Author Contributions}

A.Q., E.M., and J.S. designed the experiment and the study. J.D. and C.D. synthesized the material. A.Q., E.M., J.N., and J.S. carried out the VSM measurements and analyzed the data. A.Q. and E.M. performed the XRD and SEM characterization and analyzed the corresponding data. A.Q., E.M., and E.P. conducted the XPS and its data analysis. M.O.L., M.B., and A.W. characterized the samples by VE-PAS, cDB-PAS, and VEPALS, and analyzed the data. V.S. prepared the cross-section lamellae for TEM. A.Q., E.M., and J.N. performed the HRTEM and EFTEM and the corresponding data analysis. P.T., S.E., and F.P. carried out the EELS characterization and analyzed the data. A.Q., P.M., D.A.G., and K.L. designed the VSM 
sample holder to carry out magnetic measurements under voltage application. All authors discussed the results and commented on the article. The article was written by A.Q. and E.M.

\section{Notes}

The authors declare no competing financial interest.

\section{ACKNOWLEDGMENTS}

Financial support by the European Research Council (SPINPORICS 2014-Consolidator Grant, Agreement No. 648454), the Spanish Government (Projects MAT2017-86357-C3-1-R and associated FEDER), the Generalitat de Catalunya (2017SGR-292) and the European Union's Horizon 2020 research and innovation programme under the Marie Skłodowska-Curie Grant Agreement No. 665919 is acknowledged. E.P. is grateful to MINECO for the "Ramón y Cajal" contract (RYC-201210839). The ICN2 is funded by the CERCA programme/ Generalitat de Catalunya. ICN2 also acknowledges the support from the Severo Ochoa Program (MINECO, Grant SEV-20130295). Work at UCD is supported by the US NSF (DMR1610060 and ECCS-1611424).

\section{REFERENCES}

(1) Dieny, B.; Sousa, R. C.; Herault, J.; Papusoi, C.; Prenat, G.; Ebels, U.; Houssameddine, D.; Rodmacq, B.; Auffret, S.; BudaPrejbeanu, L. D.; Cyrille, M. C.; Delaet, B.; Redon, O.; Ducruet, C.; Nozieres, J.-P.; Prejbeanu, I. L. Spin-Transfer Effect and its Use in Spintronic Components. Int. J. Nanotechnol. 2010, 7, 591-614.

(2) Hu, J.-M.; Li, Z.; Chen, L.-Q.; Nan, C.-W. High-Density Magnetoresistive Random Access Memory Operating at Ultralow Voltage at Room Temperature. Nat. Commun. 2011, 2, 553.

(3) Shiota, Y.; Nozaki, T.; Bonell, F.; Murakami, S.; Shinjo, T.; Suzuki, Y. Induction of Coherent Magnetization Switching in a Few Atomic Layers of FeCo Using Voltage Pulses. Nat. Mater. 2012, 11, $39-43$.

(4) Bader, S. D.; Parkin, S. S. P. Spintronics. Annu. Rev. Condens. Matter Phys. 2010, 1, 71-88.

(5) Catalan, G.; Scott, J. F. Physics and Applications of Bismuth Ferrite. Adv. Mater. 2009, 21, 2463-2485.

(6) Eerenstein, W.; Mathur, N. D.; Scott, J. F. Multiferroic and Magnetoelectric Materials. Nature 2006, 442, 759-765.

(7) Wang, Y.; Hu, J.; Lin, Y.; Nan, C.-W. Multiferroic Magnetoelectric Composite Nanostructures. NPG Asia Mater. 2010, 2, 61-68.

(8) Liu, M.; Obi, O.; Lou, J.; Chen, Y.; Cai, Z.; Stoute, S.; Espanol, M.; Lew, M.; Situ, X.; Ziemer, K. S.; Harris, V. G.; Sun, N. X. Giant Electric Field Tuning of Magnetic Properties in Multiferroic Ferrite/ Ferroelectric Heterostructures. Adv. Funct. Mater. 2009, 19, 18261831.

(9) Wei, Y.; Gao, C.; Chen, Z.; Xi, S.; Shao, W.; Zhang, P.; Chen, G.; $\mathrm{Li}$, J. Four-State Memory Based on a Giant and Non-Volatile Converse Magnetoelectric Effect in FeAl/PIN-PMN-PT structure. Sci. Rep. 2016, 6, 30002.

(10) Weisheit, M.; Fähler, S.; Marty, A.; Souche, Y.; Poinsignon, C.; Givord, G. Electric Field-Induced Modification of Magnetism in Thin-Film Ferromagnets. Science 2007, 315, 349-351.

(11) Ovchinnikov, I. V.; Wang, K. L. Theory of Electric-FieldControlled Surface Ferromagnetic Transition in Metals. Phys. Rev. B: Condens. Matter Mater. Phys. 2009, 79, 020402 (R) .

(12) Cherifi, R. O.; Ivanovskaya, V.; Phillips, L. C.; Zobelli, A.; Infante, I. C.; Jacquet, E.; Garcia, V.; Fusil, S.; Briddon, P. R.; Guiblin, N.; Mougin, A.; Ünal, A. A.; Kronast, F.; Valencia, S.; Dkhil, B.; Barthélémy, A.; Bibes, M. Electric-Field Control of Magnetic Order Above Room Temperature. Nat. Mater. 2014, 13, 345-351.

(13) Quintana, A.; Zhang, J.; Isarain-Chávez, E.; Menéndez, E.; Cuadrado, R.; Robles, R.; Baró, M. D.; Guerrero, M.; Pané, S.; Nelson, B. J.; Müller, C. M.; Ordejón, P.; Nogués, J.; Pellicer, E.; Sort,
J. Voltage-Induced Coercivity Reduction in Nanoporous Alloy Films: A Boost Toward Energy-Efficient Magnetic Actuation. Adv. Funct. Mater. 2017, 27, 1701904.

(14) Duan, C.-G.; Velev, J. P.; Sabirianov, R. F.; Zhu, Z.; Chu, J.; Jaswal, S. S.; Tsymbal, E. Y. Surface Magnetoelectric Effect in Ferromagnetic Metal Films. Phys. Rev. Lett. 2008, 101, 137201.

(15) Bi, C.; Liu, Y.; Newhouse-Illige, T.; Xu, M.; Rosales, M.; Freeland, J. W.; Mryasov, O.; Zhang, S.; te Velthuis, S. G. E.; Wang, W. G. Reversible Control of Co Magnetism by Voltage-Induced Oxidation. Phys. Rev. Lett. 2014, 113, 267202.

(16) Song, C.; Cui, B.; Li, F.; Zhou, X.; Pan, F. Recent Progress in Voltage Control of Magnetism: Materials, Mechanisms, and Performance. Prog. Mater. Sci. 2017, 87, 33-82.

(17) Bauer, U.; Yao, L.; Tan, A. J.; Agrawal, P.; Emori, S.; Tuller, H. L.; van Dijken, S.; Beach, G. S. D. Magneto-Ionic Control of Interfacial Magnetism. Nat. Mater. 2015, 14, 174-181.

(18) Gilbert, D. A.; Grutter, A. J.; Arenholz, E.; Liu, K.; Kirby, B. J.; Borchers, J. A.; Maranville, B. B. Structural and Magnetic Depth Profiles of Magneto-Ionic Heterostructures Beyond the Interface Limit. Nat. Commun. 2016, 7, 12264.

(19) Gilbert, D. A.; Olamit, J.; Dumas, R. K.; Kirby, B. J.; Grutter, A. J.; Maranville, B. B.; Arenholz, E.; Borchers, J. A.; Liu, K. Controllable Positive Exchange Bias via Redox-Driven Oxygen Migration. Nat. Commun. 2016, 7, 11050.

(20) Zhang, L.; Zeng, S.; Yin, X.; Asmara, T. C.; Yang, P.; Han, K.; Cao, Y.; Zhou, W.; Wan, D.; Tang, C. S.; Rusydi, A.; Ariando; Venkatesan, T. The Mechanism of Electrolyte Gating on High- $\mathrm{T}_{\mathrm{C}}$ Cuprates: The Role of Oxygen Migration and Electrostatics. ACS Nano 2017, 11, 9950-9956.

(21) Lu, N.; Zhang, P.; Zhang, Q.; Qiao, R.; He, Q.; Li, H.-B.; Wang, Y.; Guo, J.; Zhang, D.; Duan, Z.; Li, Z.; Wang, M.; Yang, S.; Yan, M.; Arenholz, E.; Zhou, S.; Yang, W.; Gu, L.; Nan, C.-W.; Wu, J.; et al. Electric-Field Control of Tri-State Phase Transformation with a Selective Dual-Ion Switch. Nature 2017, 546, 124-128.

(22) Dubuis, G.; Yacoby, Y.; Zhou, H.; He, X.; Bollinger, A. T.; Pavuna, D.; Pindak, R.; Božović, I. Oxygen Displacement in Cuprates under Ionic Liquid Field-Effect Gating. Sci. Rep. 2016, 6, 32378.

(23) Jeong, J.; Aetukuri, N.; Graf, T.; Schladt, T. D.; Samant, M. G.; Parkin, S. S. P. Suppression of Metal-Insulator Transition in $\mathrm{VO}_{2}$ by Electric Field-Induced Oxygen Vacancy Formation. Science 2013, $339,1402-1405$.

(24) Jeong, J.; Aetukuri, N. B.; Passarello, D.; Conradson, S. D.; Samant, M. G.; Parkin, S. S. P. Giant Reversible, Facet-Dependent, Structural Changes in A Correlated-Electron Insulator Induced By Ionic Liquid Gating. Proc. Natl. Acad. Sci. U. S. A. 2015, 112, 10131018.

(25) Walter, J.; Wang, H.; Luo, B.; Frisbie, C. D.; Leighton, C. Electrostatic versus Electrochemical Doping and Control of Ferromagnetism in Ion-Gel-Gated Ultrathin $\mathrm{La}_{0.5} \mathrm{Sr}_{0.5} \mathrm{CoO}_{3-\delta}$. ACS Nano 2016, 10, 7799-7810.

(26) Kim, H. K. D.; Schelhas, L. T.; Keller, S.; Hockel, J. L.; Tolbert, S. H.; Carman, G. P. Magnetoelectric Control of Superparamagnetism. Nano Lett. 2013, 13, 884-888.

(27) Molinari, A.; Hahn, H.; Kruk, R. Voltage-Controlled On/Off Switching of Ferromagnetism in Manganite Supercapacitors. Adv. Mater. 2018, 30, 1703908.

(28) Dasgupta, S.; Das, B.; Li, Q.; Wang, D.; Baby, T. T.; Indris, S.; Knapp, M.; Ehrenberg, H.; Fink, K.; Kruk, R.; Hahn, H. Toward Onand-Off Magnetism: Reversible Electrochemistry to Control Magnetic Phase Transitions in Spinel Ferrites. Adv. Funct. Mater. 2016, 26, $7507-7515$.

(29) Zhang, Q.; He, X.; Shi, J.; Lu, N.; Li, H.; Yu, Q.; Zhang, Z.; Chen, L.-Q.; Morris, B.; Xu, Q.; Yu, P.; Gu, L.; Jin, K.; Nan, C.-W. Atomic-Resolution Imaging of Electrically Induced Oxygen Vacancy Migration and Phase Transformation in $\mathrm{SrCoO}_{2.5-\Sigma}$. Nat. Commun. 2017, 8, 104.

(30) Dendooven, J.; Deduytsche, D.; Musschoot, J.; Vanmeirhaeghe, R. L.; Detavernier, C. Conformality of $\mathrm{Al}_{2} \mathrm{O}_{3}$ and AlN Deposited by 
Plasma-Enhanced Atomic Layer Deposition. J. Electrochem. Soc. 2010, 157, G111-G116.

(31) Xie, Q.; Jiang, Y.-L.; et al. Atomic Layer Deposition of $\mathrm{TiO}_{2}$ from Tetrakis-dimethyl-amido Titanium or $\mathrm{Ti}$ Isopropoxide Precursors and $\mathrm{H}_{2} \mathrm{O}$. J. Appl. Phys. 2007, 102, 083521.

(32) Quintana, A.; Menéndez, E.; Isarain-Chávez, E.; Fornell, J.; Solsona, P.; Fauth, F.; Baró, M. D.; Nogués, J.; Pellicer, E.; Sort, J. Tunable Magnetism in Nanoporous CuNi Alloys by Reversible Voltage-Driven Element-Selective Redox Processes. Small 2018, 14, 1704396.

(33) Casas-Cabanas, M.; Binotto, G.; Larcher, D.; Lecup, A.; Giordani, V.; Tarascon, J.-M. Defect Chemistry and Catalytic Activity of Nanosized $\mathrm{Co}_{3} \mathrm{O}_{4}$. Chem. Mater. 2009, 21, 1939-1947.

(34) Gawali, S. R.; Gandhi, A. C.; Gaikwad, S. S.; Pant, J.; Chan, T.S.; Cheng, C.-L.; Ma, Y.-R.; Wu, S. Y. Role of Cobalt Cations in Short Range Antiferromagnetic $\mathrm{Co}_{3} \mathrm{O}_{4}$ Nanoparticles: A Thermal Treatment Approach to Affecting Phonon and Magnetic Properties. Sci. Rep. 2018, 8, 249.

(35) Chen, X.; Zhu, X.; Xiao, W.; Liu, G.; Feng, Y. P.; Ding, J.; Li, R.-W. Nanoscale Magnetization Reversal Caused by Electric FieldInduced Ion Migration and Redistribution in Cobalt Ferrite Thin Films. ACS Nano 2015, 9, 4210-4218.

(36) Chen, Z.; Duan, Z.; Wang, Z.; Liu, X.; Gu, L.; Zhang, F.; Dupuis, M.; Li, C. Amorphous Cobalt Oxide Nanoparticles as Active Water-Oxidation Catalysts. Chem CatChem 2017, 9, 3641-3645.

(37) Du, P.; Kokhan, O.; Chapman, K. W.; Chupas, P. J.; Tiede, D. M. Elucidating the Domain Structure of the Cobalt Oxide Water Splitting Catalyst by X-ray Pair Distribution Function Analysis. J. Am. Chem. Soc. 2012, 134, 11096-11099.

(38) Son, J. Y.; Shin, Y.-H. Direct Observation of Conducting Filaments on Resistive Switching of $\mathrm{NiO}$ Thin Films. Appl. Phys. Lett. 2008, 92, 222106.

(39) George, G.; Anandhan, S. Structural Characterization of NanoCrystalline $\mathrm{Co}_{3} \mathrm{O}_{4}$ Ultra-Fine Fibers Obtained by Sol-Gel Electrospinning. J. Sol-Gel Sci. Technol. 2013, 67, 256-266.

(40) Zasada, F.; Piskorz, W.; Sojka, Z. Cobalt Spinel at Various Redox Conditions: DFT $+U$ Investigations into the Structure and Surface Thermodynamics of the (100) Facet. J. Phys. Chem. C 2015, 119, 19180-19191.

(41) Riedel, S.; Kaupp, M. The Highest Oxidation States of the Transition Metal Elements. Coord. Chem. Rev. 2009, 253, 606-624.

(42) Dasgupta, S.; Das, B.; Knapp, M.; Brand, R. A.; Ehrenberg, H.; Kruk, R; Hahn, H. Intercalation-Driven Reversible Control of Magnetism in Bulk Ferromagnets. Adv. Mater. 2014, 26, 4639-4640.

(43) Anwand, W.; Brauer, G.; Butterling, M.; Kissener, H. R.; Wagner, A. Design and Construction of a Slow Positron Beam for Solid and Surface Investigations. Defect Diffus. Forum 2012, 331, 2540.

(44) Krause-Rehberg, R.; Leipner, H. Positron Annihilation in Semiconductors; Springer: Berlin, 1999.

(45) Khan, E. H.; Weber, M. H.; McCluskey, M. D. Formation of Isolated $\mathrm{Zn}$ Vacancies in $\mathrm{ZnO}$ Single Crystals by Absorption of Ultraviolet Radiation: A Combined Study Using Positron Annihilation, Photoluminescence, and Mass Spectroscopy. Phys. Rev. Lett. 2013, 111, 017401 .

(46) Wagner, A.; Anwand, W.; Attallah, A. G.; Dornberg, G.; Elsayed, M.; Enke, D.; Hussein, A. E. M.; Krause-Rehberg, R.; Liedke, M. O.; Potzger, K.; Trinh, T. T. Positron Annihilation Lifetime Spectroscopy at a Superconducting Electron Accelerator. J. Phys.: Conf. Ser. 2017, 791, 012004.

(47) Olsen, J. V.; Kirkegaard, P.; Pedersen, N. J.; Eldrup, M. PALSfit: A New Program for the Evaluation of Positron Lifetime Spectra. Phys. Status Solidi C 2007, 4, 4004-4006.

(48) Tuomisto, F.; Makkonen, I. Defect Identification in Semiconductors with Positron Annihilation: Experiment and Theory. Rev. Mod. Phys. 2013, 85, 1583-1631.

(49) Puska, M. J.; Nieminen, R. M. Defect Spectroscopy with Positrons: A General Calculational Method. J. Phys. F: Met. Phys. 1983, 13, 333-346.
(50) Seitsonen, A. P.; Puska, M. J.; Nieminen, R. M. Real-Space Electronic-Structure Calculations: Combination of the Finite-Difference and Conjugate-Gradient Methods. Phys. Rev. B: Condens. Matter Mater. Phys. 1995, 51, 14057-14061.

(51) Lutterotti, L.; Scardi, P. Simultaneous Structure and Size-Strain Refinement by the Rietveld Method. J. Appl. Crystallogr. 1990, 23, 246-252. 